\title{
Propagation Technique for Ultrashort Pulses II: Numerical Methods to Solve the Pulse Propagation Equation
}

\author{
Van Cao Long ${ }^{1}$, Hung Nguyen Viet ${ }^{2}$, Marek Trippenbach ${ }^{3}$, Khoa Dinh Xuan ${ }^{4}$ \\ ${ }^{1}$ Institute of Physics, University of Zielona Góra \\ Podgórna 50, 65-246 Zielona Góra, Poland \\ e-mail:V.CaoLong@if.uz.zgora.pl \\ ${ }^{2}$ Soltan Institute for Nuclear Studies \\ Hoża 69, 00-681 Warsaw, Poland \\ e-mail:hungvn@fuw.edu.pl \\ ${ }^{3}$ Department of Physics, Warsaw University \\ Hoża 69, 00-681 Warsaw, Poland \\ e-mail: matri@fuw.edu.pl \\ ${ }^{4}$ Vinh University, Nghe An, Vietnam \\ e-mail:khoadhv@yahoo.co
}

(Recived: 16 November 2007; revised: 12 May 2008; published online: 18 June 2008)

\begin{abstract}
We presented the numerical technique to approximately solve the pulse propagation equation. Two efficient methods for this problem, the Split-Step Fourier and the fourth order Runge-Kutta methods are considered. Their high accuracy are shown by comparison with analytical solutions in some particular situations. Our numerical experiments are implemented for soliton propagation and interacting high order solitons. We also numerically investigate an important technique to create ultrashort pulses, which is known as the pulse compression. It is based on high order soliton propagation in Kerr media when the effect of stimulated Raman scattering is taken into account.
\end{abstract}

Key words: propagation, ultrashort pulse, Kerr medium

\section{INTRODUCTION}

Using the Maxwell equations and Lorentz model of atoms we have derived the propagation of ultrashort pulses (of few fs) in the Kerr medium with anomalous dispersion, called Generalized Nonlinear Schrödinger Equation (GNLS) [5]:

$$
\begin{gathered}
\frac{\partial U}{\partial \xi}=\frac{i}{2} \frac{\partial^{2} U}{\partial \tau^{2}}+\delta_{3} \frac{\partial^{3} U}{\partial \tau^{3}}+ \\
+i N^{2}\left(|U|^{2} U+i S \frac{\partial}{\partial \tau}\left(|U|^{2} U\right)-\tau_{R} U \frac{\partial|U|^{2}}{\partial \tau}\right),
\end{gathered}
$$

where $U(\xi, \tau)$ is the complex envelope of the pulse. The parameters $\delta_{3}, S$ and $\tau_{R}$ govern, respectively, the effects of third-order dispersion (TOD), self-steepening and selfshift frequency. Equation (1) reduces to the Nonlinear
Schrödinger Equation (NLS) $[1,2,4]$ in the case of picosecond pulses, because then the characteristic parameters for the higher-order effects mentioned above are very small and can be neglected. NLS can be solved by the Inverse Scattering Method [2, 13, 18], but this Method cannot be applied to Eq. (1) any more. Generally it is very difficult to find analytic solutions of Eq. (1) and no such solution was known before. Only in several special cases solutions of soliton type have been obtained [17]. Nevertheless, we should note that Eq. (1) is the only one of many approximate forms for the pulse propagation equation [5, 15]. When the higher-order effects of dispersion and nonlinearity are taken into account, the pulse propagation equation becomes very complicated $[7,15,16]$ and the problem of finding a general analytic method for this equation is practically a "mission impossible". 
For these reasons several methods of finding approximate solutions of the pulse propagation equation are used. For this purpose, the numerical method is especially effective. Many algorithms of numerical calculations have been introduced by several authors $[1,3,6,7,10,12,14]$. We can generally classify them into two types, namely the finite difference methods and the quasispectrum methods [1]. The quasispectrum method is based on Fourier Transform for approximate calculations of time partial derivatives. In this manner one can reduce the problem of solving partial differential equation to solve an ordinary differential equation. By using a so called Fast Fourier Transform [1], we can drastically reduce the numerical time, so this method is intensively used in literature $[1,3,6-8,14]$.

In this paper we consider two important algorithms of quasispectrum type for the approximate calculation of solutions of the pulse propagation equation, namely the Split-Step Method and the fourth order Runge-Kutta Method. The rest of our paper is organized as follows: Section II presents the discretization rule for the pulse propagation problem in the framework of algorithms mentioned above. In Section III numerical calculations are made in some special cases for testing the accuracy of these methods. In Section IV we apply these methods to the problem of pulse compression. Section V contains conclusions.

\section{NUMERICAL METHODS}

\section{II.1. Split-Step Algorithm of second order}

In the first one we present the Split-Step Algorithm for finding approximate solutions of the pulse propagation equation. Equation (1) can be written in the following form

$$
\frac{\partial U}{\partial \xi}=(\hat{L}+\hat{N}(U)) U
$$

where $\hat{L}$ and $\hat{N}$ are the linear and nonlinear operator, respectively, acting on the envelope function:

$$
\begin{gathered}
\hat{L}=\frac{i}{2} \frac{\partial^{2}}{\partial \tau^{2}}+\delta_{3} \frac{\partial^{3}}{\partial \tau^{3}} \\
\hat{N}(U)=i N^{2}\left(|U|^{2}+i S \frac{1}{U} \frac{\partial\left(U|U|^{2}\right)}{\partial \tau}-\tau_{R} \frac{\partial|U|^{2}}{\partial \tau}\right) .
\end{gathered}
$$

Integrating Eq. (2) over the variable $\xi$ in the interval $\xi \rightarrow \xi+\Delta \xi$, we obtain [3]

$$
U(\xi+\Delta \xi, \tau)=\exp (A+B) U(\xi, \tau)
$$

with

$$
\begin{gathered}
A=\int_{\xi}^{\xi+\Delta \xi} \hat{L} d \xi^{\prime}=\hat{L} \int_{\xi}^{\xi+\Delta \xi} d \xi^{\prime}=\Delta \xi \hat{L}, \\
B=\int_{\xi}^{\xi+\Delta \xi} \hat{N}\left(U\left(\xi^{\prime}, \tau\right)\right) d \xi^{\prime} \approx \Delta \xi \hat{N}(U(\xi, \tau)) .
\end{gathered}
$$

When the step-size $\Delta \xi$ of the propagation distance is pretty small, using the Baker-Campbell-Hausdorff formula for the exponential operator involved in (5) we can present its approximate form as follows $[2,3,14,15]$ :

$$
\exp (A+B) \approx \exp \left(\frac{A}{2}\right) \exp (B) \exp \left(\frac{A}{2}\right)
$$

In this approximation we assume that operators $A$ and $B$ are commutative between themselves when $\Delta \xi$ is small. The error of the formula (8) is of the order $(\Delta \xi)^{2}$.

Substituting the expressions given above into Eq. (5) we obtain the following formula describing Split-Step algorithm for the problem (2):

$$
\begin{gathered}
U(\xi+\Delta \xi, \tau) \approx \\
\approx \exp \left(\frac{\Delta \xi}{2} \hat{L}\right) \exp (\Delta \xi \hat{N}(U(\xi, \tau))) \times \\
\times \exp \left(\frac{\Delta \xi}{2} \hat{L}\right) U(\xi, \tau) .
\end{gathered}
$$

This expression permits us to specify the approximate value of the envelope function in the location $\xi+\Delta \xi$ from its value in the $\xi$.

\section{II.2. Discrete Fourier Transform}

For calculating the value of the envelope function by (9) we should know how the action of the linear and nonlinear operators on the envelope function is calculated. Because these operators contain the time partial derivatives, one can calculate them just by Fourier Transform.

We take the value of the time variable in the finite interval $[a, b]$ which is so large that its borders do not have any influence on the final results of the calculations. We assume now the periodic condition on borders that $U(\xi, a)=U(\xi, b)$ for $\xi \in\left[0, \xi_{0}\right]$. For convenience we change the variable in (9) in such a way that it normalizes the interval $[a, b]$ into the interval $[0,2 \pi]$, and then we divide this interval into $N$ points with distance between them $\Delta \tau=2 \pi / N$. We denote these points as $\tau_{j}=2 \pi j / N$, $j=0,1,2, \ldots N$. Then we have the Discrete Fourier Transform of the series $U(\xi, \tau-j)$ as follows: 


$$
\begin{gathered}
U\left(\xi, \omega_{k}\right)=F_{k}\left[U\left(\xi, \tau_{j}\right)\right]= \\
=\frac{1}{N} \sum_{j=0}^{N-1} U\left(\xi, \tau_{j}\right) \exp \left(-i \omega_{k} \tau_{j}\right), \\
-\frac{N}{2} \leq \omega_{k} \leq \frac{N}{2}-1 .
\end{gathered}
$$

The Inverse Fourier Transform is defined as follows:

$$
\begin{gathered}
U\left(\xi, \tau_{j}\right)=F_{j}^{-1}\left[U\left(\xi, \omega_{k}\right)\right]=\sum_{k=-N / 2}^{N / 2-1} U\left(\xi, \omega_{k}\right) \exp \left(i \omega_{k} \tau_{j}\right), \\
j=0,1,2, \ldots, N .
\end{gathered}
$$

$F$ here denotes Fourier Transform and $F^{-1}$ means its inverse transform. Calculations in (10) and (11) are made effective by the fast algorithm FFT [11]. The time partial derivatives of the envelope function in both the linear and nonlinear operator (3) and (4) can be easily calculated by multiplying the Fourier coefficients $U\left(\xi, \omega_{k}\right)$ by powers of $-i \omega_{k}$ corresponding to the order of derivative and then taking the Inverse Fourier Transform. For example, the second-order derivative of the envelope function in the point $\left(\xi, \tau_{j}\right)$ can be calculated as $F_{j}^{-1}\left[-\omega_{k}^{2} F_{k}\left[U\left(\xi, \tau_{j}\right)\right]\right]$.

\section{II.3. The fourth order Runge-Kutta algorithm}

Equation (1) can also be approximately solved by using the Runge-Kutta algorithm. In this method the time discretization and calculations of time partial derivatives are the same as in the previous subsection, but the spatial derivatives are calculated by Runge-Kutta algorithm. We apply here the fourth order Runge-Kutta algorithm, very popular for solving the differential equations $[6,7,11,12]$.

After using Fourier Transform for calculating the time partial derivatives as above, equation (1) becomes

$$
\begin{aligned}
\frac{d}{d \xi}(F[U]) & =\left((-i \omega)^{2} \frac{i}{2}+(-i \omega)^{3} \delta_{3}\right) F[U]+ \\
+ & i N^{2}\left[(1+i S(-i \omega)) F\left[|U|^{2} U\right]+\right. \\
& \left.-\tau_{R} F\left[U F^{-1}\left[(-i \omega) F\left[|U|^{2}\right]\right]\right]\right] .
\end{aligned}
$$

Denoting

$$
V=\exp \left(\left(\frac{i \omega^{2}}{2}-i \omega^{3} \delta_{3}\right) \xi\right) F[U]
$$

we can rewrite (1) in the form

$$
\frac{d V}{d \xi}=f(\xi, U)
$$

where

$$
\begin{gathered}
f(\xi, U)=i N^{2} \exp \left(\left(\frac{i \omega^{2}}{2}-i \omega^{3} \delta_{3}\right) \xi\right) \times \\
\times\left[(1+S \omega) F\left[|U|^{2} U\right]+\tau_{R} F\left[U F^{-1}\left[(i \omega) F\left[|U|^{2}\right]\right]\right]\right] .
\end{gathered}
$$

Using the fourth order Runge-Kutta algorithm for Eq. (14) we calculate the value of the function $V$ in the location $\xi+\Delta \xi[11]$

$$
V(\xi+\Delta \xi)=V(\xi)+\frac{1}{6}\left[K_{1}+2\left(K_{2}+K_{3}\right)+K_{4}\right],
$$

where the coefficients $K_{i}$ are calculated as follows

$$
\begin{gathered}
K_{1}=\Delta \xi . f(\xi, U(\xi, \tau)), \\
K_{2}=\Delta \xi . f\left(\xi+\frac{\Delta \xi}{2}, U(\xi, \tau)+\frac{1}{2} K_{1}\right), \\
K_{3}=\Delta \xi . f\left(\xi+\frac{\Delta \xi}{2}, U(\xi, \tau)+\frac{1}{2} K_{2}\right), \\
K_{4}=\Delta \xi . f\left(\xi+\Delta \xi, U(\xi, \tau)+K_{3}\right) .
\end{gathered}
$$

From (13) and (16) we obtain the value of the envelope function in the location $\xi+\Delta \xi$ :

$$
\begin{gathered}
U(\xi+\Delta \xi)= \\
=F^{-1}\left[V(\xi+\Delta \xi) \exp \left(\left(-\frac{i \omega^{2}}{2}+i \omega^{3} \delta_{3}\right)(\xi+\Delta \xi)\right)\right] .
\end{gathered}
$$

Errors in applying (21) are of orders $(\Delta \xi)^{5}$. In comparison to calculations performed by (9), formula (21) has a higher accuracy, although the computational time is longer because the number of calculation steps in (15) and (20) is very large.

In the simulations performed below we have used both algorithms presented above and compared the obtained results. They are almost the same when the interval $\Delta \xi$ is relatively small.

\section{RESULTS OF NUMERICAL CALCULATIONS}

\section{III.1. Optical Solitons}

We compare in the first the numerical simulations performed by using algorithms introduced above with analytical results obtained in some spacial cases. We test in this 

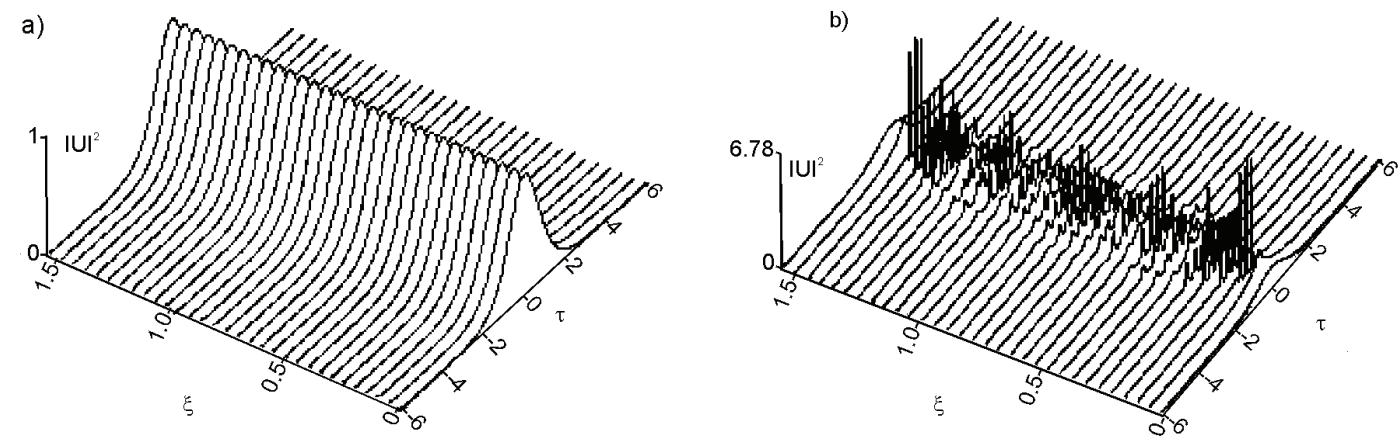

Fig. 1. Change of the pulse intensity in the propagation process for the case of fundamental (a) and tenth-order solitons (b) over one soliton period $\xi=\pi / 2$

way the accuracy of these numerical algorithms. As until now we do not know any analytical solution of the GNLS equation in literature, we will compare our results with the results of the NLS equation for the case of picosecond pulses. We consider very important phenomenon: propagation of the solitons $[1,4]$.

According to the Inverse Scattering Transform Method, when the higher-order parameters $\delta_{3}, S$ and $\tau_{R}$ in Eq. (1) equal zero and the initial shape of the pulses is the function of secant hyperbolic form, the equation will have the soliton solutions $[13,18]$. These solitons exhibit the periodic feature with a characteristic period during propagation. Except the case of the first-order (temporal) soliton (called the fundamental soliton) when the amplitude of the envelope function remains unchanged during propagation, higher-order solitons change in shape and spectrum in a complicate manner, but their shape follows a periodic pattern so that the input shape is recovered at the propagation period $\xi=\pi / 2$. The order of soliton is determined by the parameter $N$ in (1). When the value of $N$ is larger (higher-order solitons), the envelope changes in a more complicated way over one soliton period.

We simulated the pulse evolution for the first-order and tenth-order $(N=10)$ solitons over one soliton period with the input pulse having an initial amplitude [1]:

$$
U(0, \tau)=N \operatorname{sech}(\tau)
$$

Figure 1 shows these results by plotting the pulse intensity $|U(\xi, \tau)|^{2}$.

In Figure 1(a) the envelope function of the pulse has an unchanged shape in the propagation process conserving the initial form (22). In Figure 1(b) the envelope function has a complex evolution in propagation, but in the end of the period it comes back to the initial shape and this process repeats in the next periods. These results are in good agreement with analytical predictions about the periodic feature in the evolution of the envelope function. Analytical expressions for the higher-order solitons are very complicated and only in the case of the second- and third-order they are explicitly given in literature $[4,13,18]$, but for the tenth-order soliton considered above it is usually presented only by numerical results.

\section{III.2. Soliton Collisions}

In the further part of the present paper we consider the case of multiple soliton propagation. The input amplitude for a soliton pair entering the medium is expressed by

$$
U(0, \tau)=\operatorname{sech}\left(\tau-\tau_{1}\right)+r \operatorname{sech}\left[r\left(\tau+\tau_{2}\right)\right] \exp (i \theta),
$$

where $r$ is the relative amplitude of the two solitons, and $\theta$ is the relative phase between them $[1,4,7,8]$. Analytical results $[13,18]$ show that neighboring solitons either come closer or move apart because of the nonlinear interaction between them. The time of soliton collisions strongly depends on both the relative phase $\theta$ and the amplitude ratio $r$. Solitons collide periodically along the distance of propagation, the collision period usually is much greater than the soliton period. After the collision the shape of the wave amplitudes remain unchanged and stable. This effect is similar to the collision of the rigid particles, so the name "soliton" reflects the particle feature of the nonlinear waves $[1,4]$.

The following calculations are performed for the collision between the fundamental solitons and the higher-order solitons. The parameters in (23) are chosen as $r=1, \theta=0$ (equal-amplitude and in-phase case) and $\tau_{1}=\tau_{2}$ (initial spacing). Numerical results are displayed in Fig. 3. 
a)

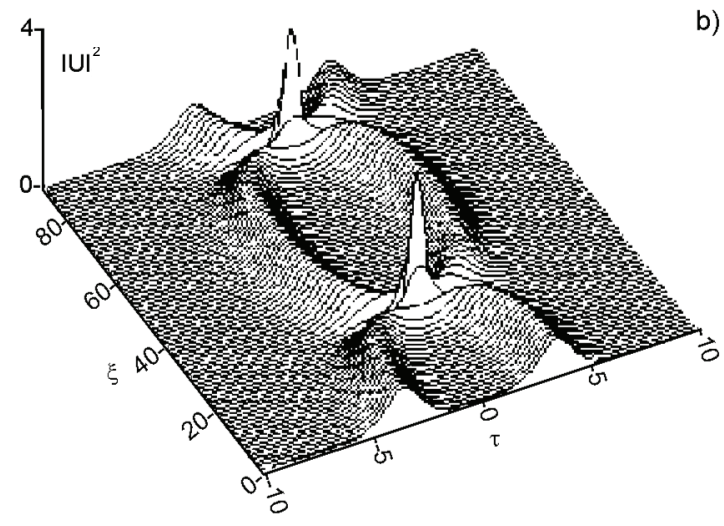

b)

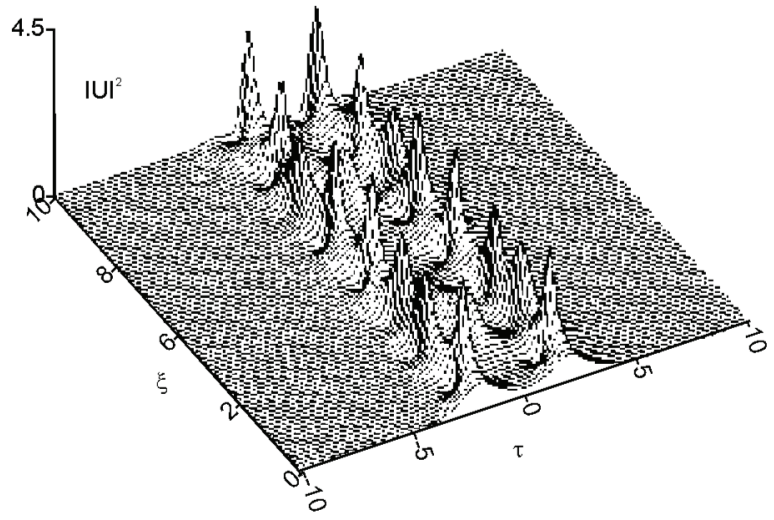

Fig. 2. Collision between two fundamental solitons over the propagation distance $\xi=90$ (a) and between two second-order solitons over the propagation distance $\xi=10$ (b)

a)

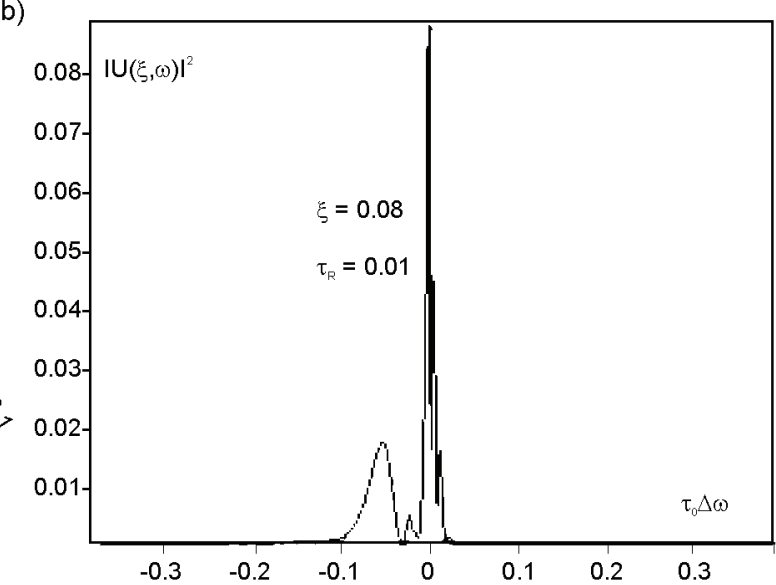

Fig. 3. Propagation of the hyperbolic secant pulse with the parameter $N=10$ over the distance $\xi=0.1$ when the stimulated Raman scattering is taken into account

Figure 2(a) displays the collision process between two fundamental solitons, where $\tau_{1}=\tau_{2}=3.5$ and the propagation distance $\xi=90$. During the propagation, firstly the two solitons attract each other, then approach one another with the increasing intensity in a location where two soliton are nearest themselves, the intensity is four times greater than the initial value. After that, the solitons repel each other and their spacing increases with distance, and the intensity decreases to the initial value. This process of attracting and repelling is repeated periodically because of the dispersion and nonlinear effects, and after each such collision the shape of the envelope function remains unchanged. In Figure 2(b) we consider the collision of two second-order solitons. The values of the parameters are chosen as $\tau_{1}=\tau_{2}=2$ and the propagation distance $\xi=10$. Because the distance between two solitons is smaller than in the previous case, the time collision is much faster. The solitons attract and repel each other in the same periodical manner but the change of the envelope function is more complicated. We also perform similar numerical calculations for the higher-order solitons and obtained results show that the complexity of the envelope function change increases with the order of the solitons. These observations are in good agreement with the calculations in $[4,8]$.

\section{APPLICATION OF THE NONLINEAR EFFECT OF STIMULATED RAMAN SCATTERING TO THE PULSE COMPRESSION}

In this part of our paper we present a very important method for creating the femtosecond pulses from the picosecond pulses of great power by stimulated Raman scattering (called the self-frequency shift) $[1,5]$.

It is clear from Fig. 1(b) that in one period the tenthorder soliton changes in a complicate manner. In some 
locations the envelope function splits to several parts with the time widths much smaller than the width of the input pulse. For this reason the propagation process is described by the NLS equation only for the pulses of great power with the width of tens or hundreds of picoseconds. When the pulse widths 1 ps or shorter, in some locations the pulse splits to the subpulses with the widths of tens or hundreds of femtoseconds during propagation. Then the higher-order dispersion and nonlinearity effects can not be neglected [5], the pulse changes in a more complicated way and the pulse propagation process should be described by the GNLS equation (1) instead of the NLS equation.

It has been recognized in [5] that for the $\mathrm{SiO}_{2}$ as a medium, in the propagation of the pulses with the widths $1 \mathrm{ps}$ or shorter, the self-frequency shift dominates over the TOD and the self-steepening. For example, if the input pulse has the width of $1 \mathrm{ps}$ and the carrier wave has the wavelength $\lambda_{0} \approx 1.55 \mu \mathrm{m}$, we have the following values of the higherorder parameters [9] $\delta_{3} \approx 0.0015, S=0.0026$ and $\tau_{R} \approx 0.01$, so the self-shift frequency dominates evidently. Under the influence of this effect the pulse is time compressed and its width decreases to a lower value. This phenomenon is used in practice in creating the ultrashort pulses. It is possible to choose an appropriate propagation distance for obtaining an ultrashort pulse with a given width. We illustrate the fact discussed above by numerical calculations for the propagation of the hyperbolic secant input pulse with the width of $1 \mathrm{ps}$, the power is characterized by the value of the parameter $N=10$, the TOD and the self-steepening parameters are neglected and $\tau_{R} \approx 0.01$. The propagation distance is $\xi=0.1$. The results are shown in Fig. 3.

Figure 3(a) displays the intensity change of the pulse. A important difference in the comparison with Fig. 1(b) is that the pulse change is more complicated here. Because of the delayed Raman response of the medium [5] the envelope function of the pulse loses its symmetrical feature and continuously shifts to the later times. In the Figure we can see that beginning from the location $\xi \approx 0.06$ the pulse splits to small pulses, its energy concentrates in the main peak and this peak is compressed further during the propagation. In the location $\xi \approx 0.09$ this part is mostly compressed and becomes a very narrow pulse with the width which is 90 times smaller than the width of the initial pulse [9] i.e. in the domain of femtoseconds. During the compression the pulse spectrum becomes much broader in comparison with the initial pulse. This fact is illustrated in Fig. 3(b). In the location $\xi=0.08$ the spectrum shifts down to the low-frequency domain. This effect is explained by the fact that Stokes processes have higher probability than anti-Stokes one, as we have considered in detail in [5].
Thus one can create the ultrashort pulses from the short pulses by pulse compression. We can construct the pulse with an arbitrary width by choosing an appropriate propagation distance. The quantity $\xi$ is normalized so that it is undimensional. In practice with the silica medium, when the carrier wave has the wavelength $\lambda_{0} \approx 1.55 \mu \mathrm{m}$, the second-order dispersion coefficient has the value $\beta^{\prime \prime}\left(\lambda_{0}\right) \approx-20 \mathrm{ps}^{2} / \mathrm{km}$, if the input pulse has $\tau_{0} \approx 1 \mathrm{ps}$, from the calculations in [5] we can predict that the appropriate length of the medium should be chosen as $1.8 \mathrm{~m}$.

\section{CONCLUSIONS}

In this paper we considered the Split-Step and four order Runge-Kutta algorithms for solving the pulse propagation equation. Calculations performed in some special cases showed good accuracy of these methods. In the next paper [19] we will apply these algorithms to investigate the propagation of the femtosecond pulses.

\section{References}

[1] G. P. Agrawal, Nonlinear Fiber Optics, Academic, San Diego, 2003.

[2] Cao Long Van, Dinh Xuan Khoa and Marek Trippenbach, Introduction to Nonlinear Optics, Vinh 2003.

[3] U. Bandelow, A. Demircan and M. Kesting, Simulation of Pulse Propagation in Nonlinear Optical Fibers, WIAS, 2003.

[4] Y. S. Kivshar and G. P. Agrawal, Optical Solitons: From Fibers to Photonic Crystals, Academic Press, San Diego, 2003.

[5] Cao Long Van, Nguyen Viet Hung, Marek Trippenbach and Dinh Xuan Khoa, Propagation technique for ultrashort pulses $I$.

[6] P. L. Francois, J. Opt. Soc. Am. B8, 276-293 (1991).

[7] G. M. Muslu and H. A. Erbay, Mathematics and Computers in Simulation 67, 581-595 (2005).

[8] M. S. Ozyaici, J. Optoelectronics and Advanced Materials 6, 71-76 (2004).

[9] G. P. Agrawal, Opt. Lett. 15, 224-226 (1990).

[10] G. H. Shiraz, P. Shum and N. Nagata, IEEE J. Quantum Electron. 31, 190- 200 (1995).

[11] W. H. Press, S. A. Teukolsky, W. T. Vetterling and B. P. Flannery, Numerical Recipes in Fortran 77 - The Art of Scientific Computing, Cambridge University Press, 1992.

[12] J. D. Hoffman, Numerical Methods for Engineers and Scientists, Marcel Dekker, 2001.

[13] L. Debnath, Nonlinear partial differential equations for scietists and engineers, Birkhauser, 1997.

[14] T. Hohage and F. Schmidt, On the Numerical Solution of Nonlinear Schrödinger Type Equations in Fiber Optics, Berlin, 2002.

[15] Cao Long Van, Marek Trippenbach, Dinh Xuan Khoa, Nguyen Viet Hung and Phan Xuan Anh, Conference on 
Theoretical Physics, Sam Son, Vietnam, 12-14 August 2003; Journal of Science, Vinh University 1A, 50 (2003).

[16] R. S. Tasgal, Y. B. Band, private communication, 2003.

[17] M. Gedalin, T. C. Scott and Y. B. Band, Phys. Rev. Lett. 78, 448-451 (1997)
[18] A. L. Maimistov and A. M. Basharov, Nonlinear optical waves, Kluwer Academic, 1999.

[19] Cao Long Van, Nguyen Viet Hung, Marek Trippenbach, Dinh Xuan Khoa, Propagation technique for ultrashort pulses III
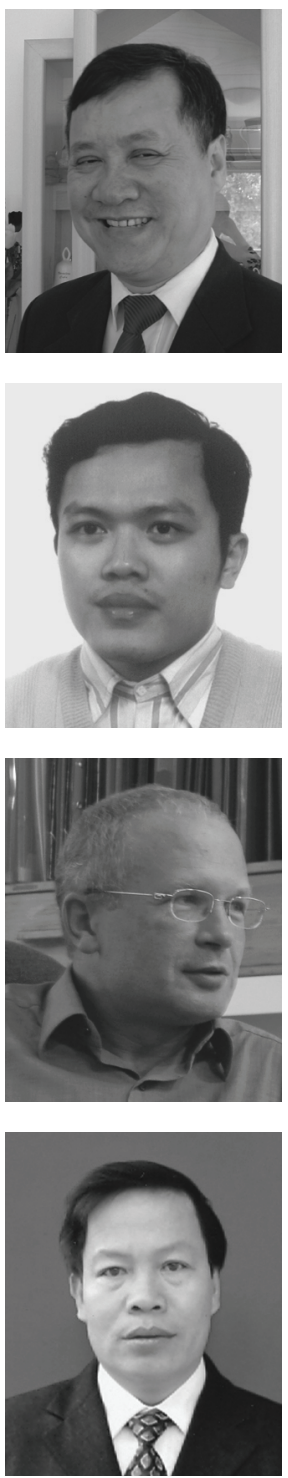

CAO LONG VAN was born in 1952 in Hanoi, Vietnam. He graduated from Warsaw University in 1976 with an MSc degree in theoretical physics. His master thesis was entitled "Callan-Symanzik Equation and Transition Probabilities" and was written under the supervision of Prof. Iwo Białynicki-Birula. In 1979, he defended his $\mathrm{PhD}$ dissertation entitled "Phase Representation in Quantum Optics", also supervised by Prof. I. Białynicki-Birula. He habilitated in 1987 in Center of Theoretical Physics, Polish Academy of Sciences in Warsaw with a thesis entitled "Noises in Quantum Optics". His research interests concern several topics in Quantum and Nonlinear Optics.

NGUYen Viet Hung received his BSc degree from Physics department of Vinh University in 2003 by defending a thesis entitled "Fundamental effects of the interaction between Electromagnetic Field and Atoms", supervised by Prof. Dinh Xuan Khoa. He continued his education at the postgraduate department of Vinh University and, in 2005, completed his Master thesis: "Propagation of light pulses in dispersive nonlinear media" which was supervised by Prof. Cao Long Van. In 2007, he started his PhD dissertation under the supervision of Prof. Marek Trippenbach at the Soltan Institute for Nuclear Studies, Warsaw. His recent research is connected with Nonlinear Optics and Bose-Einstein condensation.

MareK TrippenbaCh, PhD, DSc, is a graduate of Warsaw University. His field of interests covers various topics of Quantum and Nonlinear Optics. His recent research is focused on solitons in such nonlinear systems as Optical Nonlinear Media and Bose-Einstein condensates. He was a co-author of the well-known experiment which was carried out by the group led by a Nobel Prize laureate from 1997: William Phillips. In this experiment, the Four-Wave Mixing of Condensates was observed. This phenomenon is considered to be the first observable effect of Nonlinear Atom Optics. During his stay in JILA (Boulder, Colorado), Marek Trippenbach worked with yet another laureate of the Nobel Prize (from 2001): Carl Wieman who was among the first to experimentally discover the Bose-Einstein Condensate. Nowadays, he is working at the Institute of Theoretical Physics, Warsaw University. He is a fellow in the British Institute of Physics.

Dinh XUAN KhoA graduated from Vinh University, Vietnam, with a major in physics in 1981. He completed a PhD course in Quantum Optics in 1996. His doctoral thesis entitled "Generative Kinetics of Dye Lasers" was supervised by Prof. Cao Long Van and Prof. Dao Xuan Hoi. His field of interests covers a large variety of topics in Quantum and Nonlinear Optics. He is exceptionally interested in Soliton Theory and research concerning long-distance optical communication systems. Recently, Prof. Dinh Xuan Khoa has been a deputy rector of Vinh University. 